\title{
Impaired Speech Repetition and Left Parietal Lobe Damage
}

\author{
Julius Fridriksson, ${ }^{1}$ Olafur Kjartansson, ${ }^{2}$ Paul S. Morgan, ${ }^{3}$ Haukur Hjaltason, ${ }^{2}$ Sigridur Magnusdottir, ${ }^{2}$ \\ Leonardo Bonilha, ${ }^{3}$ and Christopher Rorden ${ }^{1}$ \\ ${ }^{1}$ Department of Communication Sciences and Disorders, University of South Carolina, Columbia, South Carolina 29208, ${ }^{2}$ Landspitali-University Hospital, \\ IS-101 Reykjavik, Iceland, and ${ }^{3}$ Medical University of South Carolina, Charleston, South Carolina 29425
}

Patients with left hemisphere damage and concomitant aphasia usually have difficulty repeating others' speech. Although impaired speech repetition, the primary symptom of conduction aphasia, has been associated with involvement of the left arcuate fasciculus, its specific lesion correlate remains elusive. This research examined speech repetition among 45 stroke patients who underwent aphasia testing and MRI examination. Based on lesion- behavior mapping, the primary structural damage most closely associated with impaired speech repetition was found in the posterior portion of the left arcuate fasciculus. However, perfusion-weighted MRI revealed that tissue dysfunction, in the form of either frank damage or hypoperfusion, to the left inferior parietal lobe, rather than the underlying white matter, was associated with impaired speech repetition. This latter result suggests that integrity of the left inferior parietal lobe is important for speech repetition and, as importantly, highlights the importance of examining cerebral perfusion for the purpose of lesion- behavior mapping in acute stroke.

\section{Introduction}

The inability to repeat others' speech is common among stroke patients with aphasia-a language disorder usually caused by left hemisphere injury. Aphasia has many patterns and levels of severity, and although impaired repetition is not uncommon, it is the primary impairment in conduction aphasia. Wernicke predicted that severing the connection between speech production and comprehension would result in a disorder in which comprehension remained intact and speech output was fluent, but marked by speech errors (Wernicke, 1874; Lichtheim, 1885). Although Wernicke had never seen such a patient, one was later described by Lichtheim (1885). Lichtheim also recognized that such patients have difficulty repeating others' speech. Today, the definition of conduction aphasia is largely unchanged: impaired repetition with relatively spared speech fluency and auditory comprehension. It is classically viewed as resulting from brain damage involving the left arcuate fasciculus where, according to the Wernicke-Lichtheim model (Lichtheim, 1885), the temporal lobe's posterior comprehension areas are disconnected from the anterior speech production regions of the frontal lobe.

Conduction aphasia and its accompanying brain damage have been well described in the literature (e.g., Damasio and Damasio, 1980; Poncet et al., 1987; Demeurisse and Capon, 1991). Most of these studies suggest that, indeed, damage to the left arcuate fasciculus results in conduction aphasia. However, several recent papers have challenged this claim, suggesting that damage to cortical gray matter in the left inferior parietal lobe is sufficient to

Received March 4, 2010; revised June 25, 2010; accepted July 6, 2010

This work was supported by National Institute on Deafness and Other Communication Disorders Grant DC009571 (principal investigators: J.F. and C.R.).

Correspondence should be addressed to Julius Fridriksson, Department of Communication Sciences and Disorders, University of South Carolina, Columbia, SC 29208. E-mail: jriidrik@sc.edu.

DOI:10.1523/JNEUROSCI.1120-10.2010

Copyright $\odot 2010$ the authors $\quad 0270-6474 / 10 / 3011057-05 \$ 15.00 / 0$ elicit conduction aphasia (Anderson et al., 1999; Hickok et al., 2000; Bartha and Benke, 2003; Quigg et al., 2006; Geldmacher et al., 2007). The purpose of this study was to examine the critical lesion location associated with impaired speech repetition-conduction aphasia's primary symptom. Patients with acute stroke to the left hemisphere were examined at bedside using a short aphasia battery including items testing speech repetition. All patients also underwent MRI scanning to elucidate the lesion location most likely to impair speech repetition.

Our protocol extended previous studies of repetition disorders in four ways. First, previous studies have attempted to identify patients with pure conduction aphasia (i.e., patients with repetition difficulties despite relatively spared speech comprehension and fluency). Logically, this selection criterion should bias the results away from identifying regions near the primary speech centers. Therefore, our aim was to identify regions that predict repetition errors, even when other speech impairments may be present. Second, previous studies have focused on patients with repetition disorders, excluding patients with damage to the brain without repetition problems. The problem here is that a brain region may be identified either because of its relationship to the task or due to its vulnerability to injury. Including patients with and without impaired speech repetition permitted us to identify regions that are commonly injured in impaired patients, but spared in patients without this deficit. Third, examining a large number of patients made it possible to conduct an objective high-resolution voxelwise statistical analysis. Fourth, we included an analysis of acute perfusion imaging in addition to structural measures of brain imaging.

\section{Materials and Methods}

Participants. The participants included in this study were 45 consecutive stroke patients admitted to the Landspitali-University Hospital in Reykjavik, Iceland. All participants had incurred a single event ischemic stroke to the left hemisphere and provided informed consent for study inclu- 


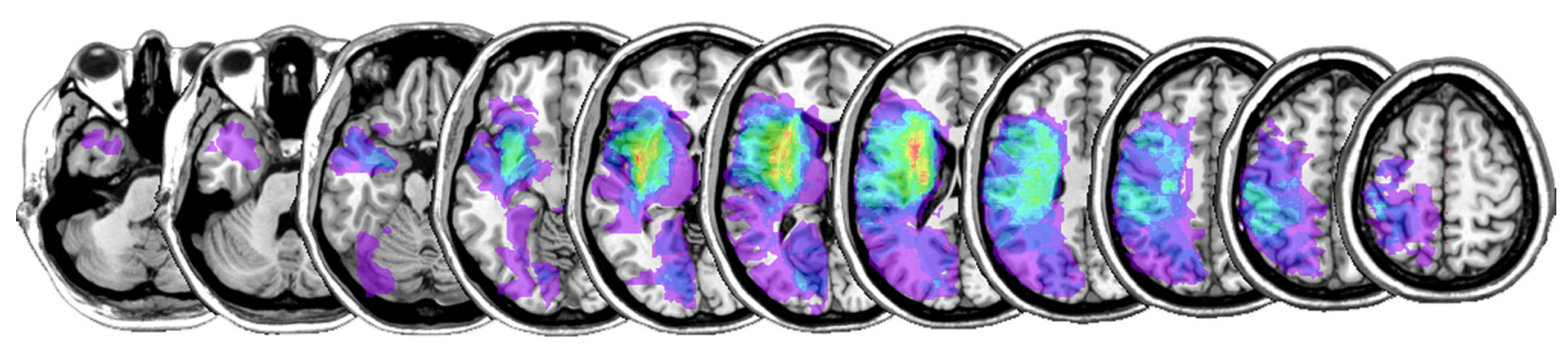

1

Figure 1. Lesion map for the 39 patients where lesions were overlaid on a standard brain template. The greatest lesion overlay was noted in anterior insula where 14 patients had damage.

Table 1. A summary of patients' performance on the BEST-2

\begin{tabular}{lcccc}
\hline & No impairment & Mild & Moderate & Severe \\
\hline 1. Conversational Expression & 7 & 17 & 6 & 9 \\
2. Naming Objects & 19 & 6 & 3 & 11 \\
3. Describing Objects & 21 & 1 & 6 & 11 \\
4. Repeating Sentences & 13 & 13 & 5 & 8 \\
5. Pointing to Objects & 6 & 22 & 4 & 7 \\
6. Pointing to Parts of Picture & 6 & 24 & 5 & 4 \\
7. Reading & 5 & 16 & 11 & 7 \\
Severity rating & 8 & 22 & 5 & 4 \\
\hline
\end{tabular}

The data represent the number of patients who scored in each severity level on the seven subtests included in the BEST-2. A tally of patients' overall severity is included in the bottom row of the table.

sion. Exclusion criteria were as follows: (1) non-native speaker of Icelandic; (2) clinical history of stroke; (3) history of dementia, alcoholism, or major psychiatric illness; and (4) contraindication for MRI examination. Six patients were excluded based on MRI-related issues (e.g., excessive movement artifacts or corrupted MRI data). Therefore, data from 39 patients were included in the final statistical analyses described below. The mean patient age was 63.5 years ( range $=34-85, \mathrm{SD}=12$ ). For most participants, behavioral and MRI examination were completed within $3 \mathrm{~d}$ of hospital admission, but in a few cases participants were examined as late as $20 \mathrm{~d}$ following stroke due to factors such as fatigue or other stroke sequelae. Behavioral and MRI examinations were always completed with $<24 \mathrm{~h}$ separating them. A lesion overlay map for the patient group is shown in Figure 1. The greatest lesion overlap was revealed in the left anterior insula (MNI coordinates: $-28,5,20)$.

Behavioral testing. All participants were administered a neuropsychological workup that included the Bedside Evaluation Screening Test, second edition (BEST-2) (West et al., 1998). The BEST-2's Repetition subtest served to quantify the severity of repetition impairment. This section includes five items where the level of presentation of each item is titrated based on patients' success with the previous item. Each item includes the following: (1) complete sentence; (2) phrase; (3) single word. Based on the BEST-2 overall aphasia severity scale, 4 patients had severe language impairment, 5 had moderate impairment, and 30 presented with mild or no language impairment. Table 1 includes a summary of the testing results from the BEST-2 for the patient group.

Neuroimaging data. All participants underwent a 1.5 T MRI workup (using a Siemens Avanto system) that included T1-weighted imaging (T1-MRI, reconstructed resolution $0.9 \times 0.9 \times 0.9 \mathrm{~mm}$ ), axial diffusionweighted imaging (DWI, $1.2 \times 1.2 \times 6.5 \mathrm{~mm})$, FLAIR $(0.72 \times 0.72 \times 6.5$ $\mathrm{mm}$ ), and contrast enhanced $\mathrm{T}_{2}{ }^{*}$ perfusion-weighted MRI (PWI, $1.8 \times$ $1.8 \times 6.5 \mathrm{~mm})$. Images were converted from DICOM to NIfTI format using dcm2nii (www.mricro.com), which preserves spatial coordinates, ensuring a good starting estimate for the subsequent coregistration.

Acute stroke brain lesions were demarcated on DWI images by a trained neurologist (L.B.) with extensive experience with lesionsymptom mapping who was blind to the individual's symptoms. The lesion maps were then transformed into stereotaxic space via spatial processing using SPM5 as follows: Initially, DWI images were coregis- tered to the individual's T1 scan. This transform was applied to the lesion map. Then, the T1-scan was coregistered to the T1 template image provided with SPM5 (offering an accurate starting estimate for the subsequent normalization). Finally, the T1 image was warped to standard stereotaxic space using SPM5's unified segmentation and normalization algorithm, which has proved robust even in the case of large lesions (Crinion et al., 2007). The resulting transforms were used to reslice both the T1 images and lesion maps to an isotropic $2 \mathrm{~mm}$ in standard stereotaxic space, enabling a voxelwise analysis of the lesion maps.

The PWI data were processed using the Java Image software (Xinapse Systems), which analyzes the time series of the MR signal modulation due to the passage of the bolus of injected contrast agent through the brain (Ostergaard et al., 1996). This produces voxelwise maps of estimates of time to peak concentration of the contrast agent (TTP). Motion correction was performed on the time series of images before calculation of the perfusion parameters, also using Java Image. Perfusion parameter maps were produced in the same space as the acquired $\mathrm{T}_{2}{ }^{*}$-weighted PWI allowing the same transform from coregistration performed using the PWI to be applied to the perfusion parameter maps. SPM5 was used to align the PWI images. First, the mean PWI image from each individual was coregistered to the "EPI.nii" template that is distributed with SPM (this image is in MNI space, and is based on T2*-weighted images that have similar contrast to the PWI). This transform was used to reslice the TTP image to standard $2 \mathrm{~mm}$ isotropic stereotaxic space.

Postprocessing statistical analyses were performed using the DWI lesion data and the PWI data. The DWI lesion data consisted of binary voxelwise maps (lesion versus nonlesioned) and were submitted to voxelwise lesion-behavior mapping (VLBM) (Bates et al., 2003). VLBM was performed using NPM (nonparametric mapping) (Rorden et al., 2007) (a software package available from www.mricro.com), via a voxel-byvoxel logistic regression. Impaired auditory comprehension is common among aphasic patients and, like speech repetition, tends to be more impaired in patients with more severe aphasia. It should be noted that speech comprehension deficits could lead to knock-on errors in speech production. Since our work was focused on speech production, we modeled the level of speech comprehension as a cofactor of no interest. Specifically, an independent $Z$-score was computed for each voxel, computing whether speech repetition performance was correlated with injury to that voxel, using the level of auditory comprehension performance as a regressor. This comprehension factor was measured as patients' ability to pair pictures of common objects with their verbally presented name (subtest nr. 5 on the BEST-2). The resulting statistical map from the VLBM analysis was corrected for multiple comparisons using a 1\% false-discovery rate (FDR) threshold (Rorden et al., 2009).

The PWI data, conversely, comprised whole-brain voxelwise maps with continuous values. Therefore, a whole-brain weighted leastsquares (WLS) regression was used to evaluate the severity of speech repetition impairment (lower scores on the BEST-2 denote more severe impairment). The results were corrected for multiple comparisons using a $1 \%$ FDR. 

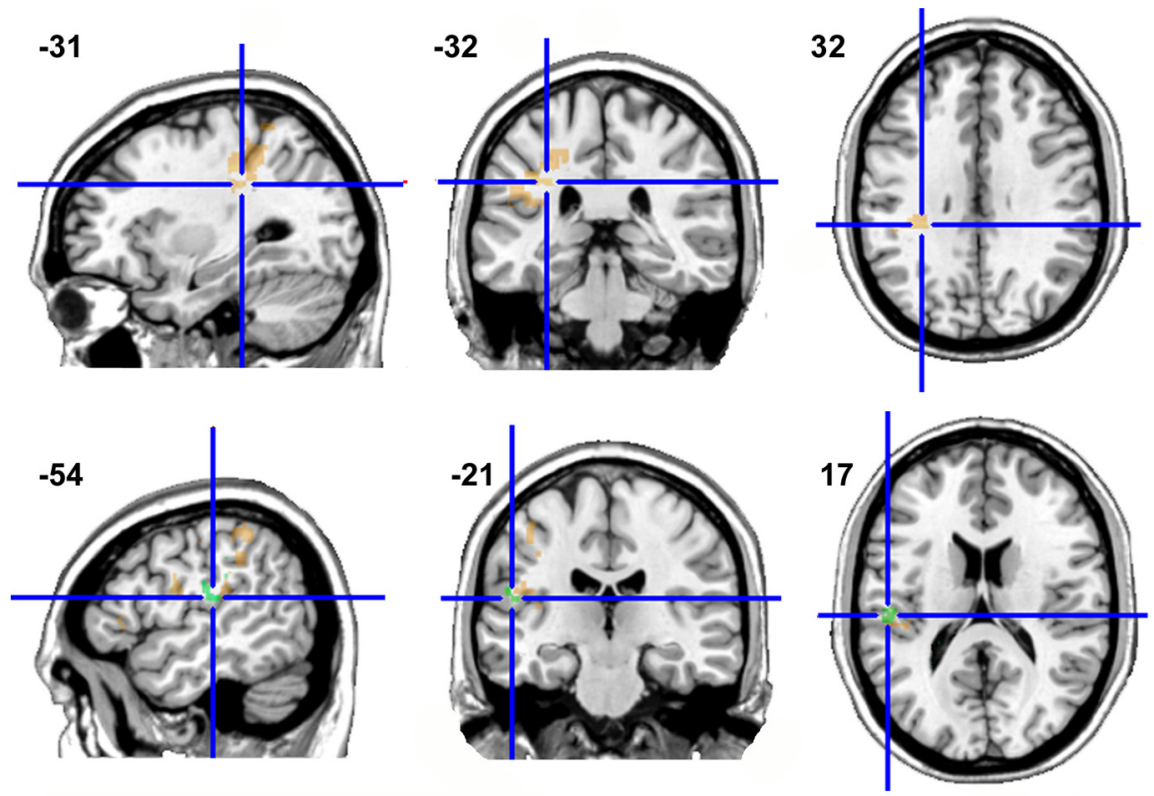$$
-5
$$

0

Figure 2. Structural brain damage (red-yellow scale) and abnormal perfusion (blue-green scale) associated with impaired speech repetition. The cross hairs denote the voxel with the highest predictive value for the structural lesion data (top panel) and the PWI data (bottom panel). The lesion maps were overlaid on a standard brain template.

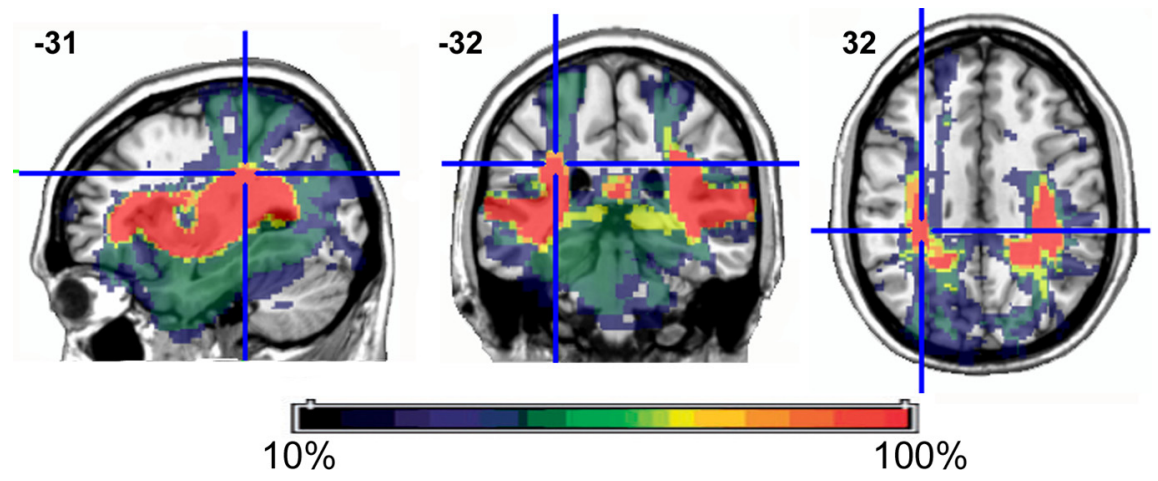

Figure 3. A probabilistic map of the arcuate fasciculus. The coordinates correspond to the voxel location revealed to have the highest predictive value of impaired speech repetition. The color scale denotes the percentage overlap for the white matter fibers among the 54 subjects.

\section{Results}

The VLBM analysis of the structural lesion data revealed that damage to white matter underlying the left supramarginal gyrus $(Z=2.8)$ is strongly related to impaired speech repetition. The voxel with the highest $Z$-score (located at coordinates $-31,-32$, 32) for this analysis is shown in the cross-hairs in Figure 2 (top panel). To emphasize that this lesion location occurred within the arcuate fasciculus the same MNI coordinates are highlighted in Figure 3 where a probabilistic map of the arcuate fasciculus was overlaid on a standard brain template. This probabilistic map was constructed from diffusion tensor imaging MRI (DTI) data in 54 older adults (see Bonilha and Fridriksson, 2009) where the white matter tracts were traced from Brodmann's area (BA) 22 to BA 45. Additional structural damage associated with impaired speech repetition was noted in Broca's area $(-41,36,10)$.

The WLS regression analysis of the PWI data revealed that the best predictor of impaired speech repetition was located in the inferior portion of the left supramarginal gyrus, $Z=$
-4.21 (Fig. 2, bottom panel). That is, patients who had high TTP values (i.e., hypoperfusion) in this area tended to score low on the repetition subtest. This analysis also revealed a cluster in the temporal-parietal junction (TPJ) $(-44$, $-35,24)$ (not pictured).

\section{Discussion}

A strong relationship between structural damage to the arcuate fasciculus and impaired speech repetition agrees with the classical view of aphasia. This suggests that a disconnection between the anterior and posterior speech areas disrupts flow of information needed both to convert the auditory speech code into motor speech production and to maintain speech monitoring based on the auditory speech code (Wernicke, 1874; Geschwind, 1965). This account is plausible given that the arcuate fasciculus is the largest intrahemispheric white matter tract connecting the critical posterior speech comprehension areas with anterior motor speech areas (Catani et al., 2005). Although the underlying speech impairment may vary in a group that includes patients with different lesion locations and language profiles, the best lesion predictor of impaired speech repetition involves the arcuate fasciculus. Speech production not only relies on the frontal motor planning and execution areas but also on feedback from the posterior auditory speech areas (Hickok and Poeppel, 2007; Tourville et al., 2008). It is of interest that damage to a "ventral" pathway between the anterior and posterior speech areas via the middle longitudinal fasciculus and extreme capsule (e.g., Parker et al., 2005) was not associated with impaired speech repetition.

As suggested by Rorden and Karnath (2004), lesion-behavior mapping is a powerful tool to study both normal and disordered brain function. However, Hillis (2007) has argued that relying only on structural data in acute stroke patients gives an incomplete picture when inferring the relationship between lesion location and behavioral impairment. Rather, acute lesionbehavior mapping can benefit from assessment of cerebral perfusion abnormalities since hypoperfusion beyond the actual lesion is commonly observed in the first hours and even days following stroke (Karonen et al., 2000). Assessment of both diffusion as well as cerebral perfusion anomalies allows for direct quantification of the frank lesion in addition to structurally intact cortical areas not receiving sufficient perfusion to function (the ischemic penumbra). Hillis et al. (2006) have combined both structural and functional data in several lesion-behavior studies. Compared to structural lesion data, PWI data highlight both abnormal brain perfusion as well as the cytotoxic lesion (as severely decreased cerebral blood flow/volume or very slow transit time of a contrast bolus). In the current study, PWI revealed somewhat different results when compared to structural lesion data; the best 
lesion predictor of impaired speech repetition was located in gray matter of the left inferior supramarginal gyrus as well as the TPJ. It is important to note here that the analysis including only the structural lesions identified a white matter area deep to the left inferior parietal lobe. Thus, it is probable that structural lesions that only involve damage to the underlying white matter also cause hypoperfusion in the overlying cortex (Hillis et al., 2002, 2004).

According to the directions into velocities model (DIVA model) of speech production, the left inferior parietal lobule includes a crucial component of the speech network (Guenther, 2006). Here, somatosensory state maps and somatosensory error maps are recruited to provide feedback for motor speech production planned and executed in Broca's area and the motor cortex, respectively. Bohland and Guenther (2006) found that increased syllable sequence complexity resulted in increased cortical activity in the anterior speech areas as well as the parietal lobe, suggesting that damage to the somatosensory component would result in impaired speech repetition and spontaneous production. Others have suggested that the inferior parietal lobe may play a role in processing the temporal order of speech syllables (Moser et al., 2009). Such an account is also consistent with the results described here, linking structural and functional damage in the inferior parietal lobe to impaired speech repetition. A study by Parker et al. (2005) revealed white matter connections between the classical speech areas and the inferior parietal lobe, further suggesting that this area may play an important role in speech processing.

In addition to the lesion to the supramarginal gyrus, damage to the TPJ was associated with impaired speech repetition. This is not surprising, given the previously discussed notion that different underlying impairments may affect speech repetition among patients with different lesion locations and speech impairments. The Dual Stream model (Hickok and Poeppel, 2007) suggests that the TPJ is important for translating the auditory speech code into articulatory code. Dhanjal et al. (2008) further refined this hypothesis and suggested that this area is important for polysensory integration during speech processing. It is straightforward to see how breakdown in this mechanism would lead to severe impairment of speech repetition, where a heard word must be integrated with motor representation for speech production. The point here is not to attribute a specific process to the areas identified in the PWI analysis; rather, the current data emphasize that among all the different cortical regions affected in this patient sample, damage to the supramarginal gyrus and the TPJ is most likely to impair speech repetition.

It is important to point out that the present study did not examine a direct relationship between localized brain damage and conduction aphasia, an aphasic disorder marked by a distinct impairment in speech repetition with relatively spared language comprehension and speech production. Patients with a wide range of aphasia types as well as patients who showed no signs of aphasia were included in this study. Thus, the study sample included some patients who had difficulty repeating due to factors such as apraxia of speech, nonfluent aphasia, or auditory comprehension impairment rather than the primary repetition impairment seen in conduction aphasia. On the other hand, as noted earlier, excluding patients with other symptoms of aphasia will necessarily bias any analysis against identifying regions near the primary language zones. Despite this caveat, the VLBM analysis for both structural and perfusion data did reveal brain damage commonly associated with conduction aphasia as being the most likely lesion predictor of impaired speech repetition. In an examination of six patients with conduction aphasia, Damasio and Damasio (1980) found that among other affected cortical regions, the arcuate fasciculus was involved in five cases. However, they reported that other areas such as the insula, the auditory complex, and the supramarginal gyrus were involved in these five cases. Baldo et al. (2008) found that among seven patients with conduction aphasia, the greatest structural lesion overlap was found in the left posterior speech areas including the superior temporal and inferior parietal lobules. Although it is tempting to suggest that the current findings corroborate others' findings regarding gray rather than white matter damage as being the culprit in conduction aphasia, difficulty with speech repetition is clearly not the only problem observed in conduction aphasia. These patients also have distinct patterns of speech production marked by frequent phonemic paraphasias and in the more severe forms of the disorder, neologisms. In addition, as originally suggested by Wernicke (1874), the patients have a distinctive ability to monitor their speech errors. The present data do not address these other features. Nevertheless, they indicate that impaired speech repetition, as the most salient feature of conduction aphasia, is strongly associated with damage to the left arcuate fasciculus, supramarginal gyrus, and TPJ.

\section{References}

Anderson JM, Gilmore R, Roper S, Crosson B, Bauer RM, Nadeau S, Beversdorf DQ, Cibula J, Rogish M 3rd, Kortencamp S, Hughes JD, Gonzalez Rothi LJ, Heilman KM (1999) Conduction aphasia and the arcuate fasciculus: a reexamination of the Wernicke-Geschwind model. Brain Lang 70:1-12.

Baldo JV, Klostermann EC, Dronkers NF (2008) It's either a cook or a baker: patients with conduction aphasia get the gist but lose the trace. Brain Lang 105:134-140.

Bartha L, Benke T (2003) Acute conduction aphasia: an analysis of 20 cases. Brain Lang 85:93-108.

Bates E, Wilson SM, Saygin AP, Dick F, Sereno MI, Knight RT, Dronkers NF (2003) Voxel-based lesion-symptom mapping. Nat Neurosci $6: 448-450$

Bohland JW, Guenther FH (2006) An fMRI investigation of syllable sequence production. Neuroimage 32:821-841.

Bonilha L, Fridriksson J (2009) Subcortical damage and white matter disconnection associated with non-fluent speech. Brain 132:e108.

Catani M, Jones DK, ffytche DH (2005) Perisylvian language networks of the human brain. Ann Neurol 57:8-16.

Crinion J, Ashburner J, Leff A, Brett M, Price C, Friston K (2007) Spatial normalization of lesioned brains: performance evaluation and impact on fMRI analyses. Neuroimage 37:866-875.

Damasio H, Damasio AR (1980) The anatomical basis of conduction aphasia. Brain 103:337-350.

Demeurisse G, Capon A (1991) Brain activation during a linguistic task in conduction aphasia. Cortex 27:285-294.

Dhanjal NS, Handunnetthi L, Patel MC, Wise RJ (2008) Perceptual systems controlling speech production. J Neurosci 28:9969-9975.

Geldmacher DS, Quigg M, Elias WJ (2007) MR tractography depicting damage to the arcuate fasciculus in a patient with conduction aphasia. Neurology 69:321.

Geschwind N (1965) Disconnexion syndromes in animals and man. Brain $88: 237-294$.

Guenther FH (2006) Cortical interactions underlying the production of speech sounds. J Commun Disord 39:350-365.

Hickok G, Poeppel D (2007) The cortical organization of speech processing. Nat Rev Neurosci 8:393-402.

Hickok G, Erhard P, Kassubek J, Helms-Tillery AK, Naeve-Velguth S, Strupp JP, Strick PL, Ugurbil K (2000) A functional magnetic resonance imaging study of the role of left posterior superior temporal gyrus in speech production: implications for the explanation of conduction aphasia. Neurosci Lett 287:156-160.

Hillis AE (2007) Magnetic resonance perfusion imaging in the study of language. Brain Lang 102:165-175.

Hillis AE, Wityk RJ, Barker PB, Beauchamp NJ, Gailloud P, Murphy K, Cooper O, 
Metter EJ (2002) Subcortical aphasia and neglect in acute stroke: the role of cortical hypoperfusion. Brain 125:1094-1104.

Hillis AE, Barker PB, Wityk RJ, Aldrich EM, Restrepo L, Breese EL, Work M (2004) Variability in subcortical aphasia is due to variable sites of cortical hypoperfusion. Brain Lang 89:524-530.

Hillis AE, Kleinman JT, Newhart M, Heidler-Gary J, Gottesman R, Barker PB, Aldrich E, Llinas R, Wityk R, Chaudhry P (2006) Restoring cerebral blood flow reveals neural regions critical for naming. J Neurosci 26: 8069-8073.

Karonen JO, Liu Y, Vanninen RL, Østergaard L, Kaarina Partanen PL, Vainio PA, Vanninen EJ, Nuutinen J, Roivainen R, Soimakallio S, Kuikka JT, Aronen HJ (2000) Combined perfusion- and diffusion-weighted MR imaging in acute ischemic stroke during the 1st week: a longitudinal study. Radiology 217:886-894.

Lichtheim L (1885) On aphasia. Brain 7:433-484.

Moser D, Baker JM, Sanchez CE, Rorden C, Fridriksson J (2009) Temporal order processing of syllables in the left parietal lobe. J Neurosci 29: $12568-12573$.

Ostergaard L, Weisskoff RM, Chesler DA, Gyldensted C, Rosen BR (1996) High resolution measurement of cerebral blood flow using intravascular tracer bolus passages. Part I: Mathematical approach and statistical analysis. Magn Reson Med 36:715-725.
Parker GJ, Luzzi S, Alexander DC, Wheeler-Kingshott CA, Ciccarelli O, Lambon Ralph MA (2005) Lateralization of ventral and dorsal auditorylanguage pathways in the human brain. Neuroimage 24:656-666.

Poncet M, Habib M, Robillard A (1987) Deep left parietal lobe syndrome: conduction aphasia and other neurobehavioural disorders due to a small subcortical lesion. J Neurol Neurosurg Psychiatry 50:709-713.

Quigg M, Geldmacher DS, Elias WJ (2006) Conduction aphasia as a function of the dominant posterior perisylvian cortex. J Neurosurg 104: 845-848.

Rorden C, Karnath HO (2004) Using human brain lesions to infer function: a relic from a past era in the fMRI age? Nat Rev Neurosci 5:813-819.

Rorden C, Karnath HO, Bonilha L (2007) Improving lesion-symptom mapping. J Cogn Neurosci 19:1081-1088.

Rorden C, Fridriksson J, Karnath HO (2009) An evaluation of traditional and novel tools for lesion behavior mapping. Neuroimage 44:1355-1362.

Tourville JA, Reilly KJ, Guenther FH (2008) Neural mechanisms underlying auditory feedback control of speech. Neuroimage 39:1429-1443.

Wernicke C (1874) Der aphasische Symptomencomplex: eine psychologische Studie auf anatomischer Basis: Cohn and Weigert.

West JF, Sands ES, Ross-Swain D (1998) Bedside evaluation screening test. Austin, TX: Pro-Ed. 